\title{
COMPOSTOS VOLÁTEIS DE TRÊS CULTIVARES DE MANGA (Mangifera indica L.) ${ }^{1}$
}

\author{
M.R.B. FRANCO ${ }^{2, *}$, D. RODRIGUEZ-AMAYA², F.M. LANÇAS ${ }^{3}$
}

\begin{abstract}
RESUMO
Os compostos voláteis de três cultivares de manga (Haden, Tommy-Atkins e Keitt) procedentes do Estado de São Paulo, foram isolados por uma técnica de headspace dinâmico, envolvendo sucção em polímero poroso. A separação da mistura complexa de voláteis foi efetuada por cromatografia gasosa de alta resolução. Alguns compostos voláteis foram identificados por cromatografia gasosa-espectrometria de massas e índices de Kovats. Os hidrocarbonetos monoterpênicos foram os compostos mais abundantes no headspace das amostras. Car-3-eno foi o componente majoritário dos cultivares Haden e Keitt, enquanto a Tommy-Atkins mostrou predominância de car-3-eno e $\alpha$-pineno. Outros compostos identificados foram $\alpha$-fencheno, $\alpha$-canfeno, $p$-cimeno, $\beta$-mirceno, $\beta$-felandreno, limoneno, $\alpha$ terpinoleno, $\beta$-cariofileno e $\alpha$-humuleno. Os três lotes analisados para cada cultivar mostraram grande homogeneidade, não havendo diferença significativa ao nivel de $5 \%$.
\end{abstract}

Palavras-chave: compostos voláteis; aroma; headspace dinâmico; manga; cultivares.

\section{SUMMARY}

VOLATILE COMPOSITION OF THREE CULTIVARS OF MANGO (Mangifera indica L.). The volatile compounds of three mango cultivars (Haden, Tommy-Atkins and Keitt), from the State of São Paulo, were isolated by a dynamic headspace technique involving suction on a porous polymer. The complex mixture of volatile compounds was separated by high-resolution gas chromatography. Some volatiles were identified by gas chromatography-mass spectrometry and Kovats Indices. The monoterpene hydrocarbons were the most abundant in the headspace of the fruits. Car-3-ene was the major component of the cultivars Haden and Keitt, while the Tommy Atkins mango exhibited predominance of two constituents, car-3-ene and $\alpha$-pinene. Other identified compounds were $\alpha$-fenchene, $\alpha$-camphene, $\mathrm{p}$ cimene, $\beta$-mircene, $\beta$-phellandrene, limonene, $\alpha$-terpinolene, $\beta$-caryophyllene e $\alpha$-humulene. Samples of the same cultivar demonstrated great homogeneity, with no statistically significant difference being observed among the three lots analyzed for each cultivar. Keywords: volatile compounds; flavour; dynamic headspace; mango; cultivars.

\section{1 - INTRODUÇÃO}

O Brasil é o sétimo maior produtor de mangas, com produção de aproximadamente um milhão de toneladas anuais, sendo o Estado de São Paulo o maior produtor nacional. O Brasil está conseguindo ampliar espaço no mercado internacional para a exportação de manga e ocupa a sexta posição entre os países exportadores. Em 1999, a exportação de 67 mil toneladas de manga rendeu 36 milhões de dólares. Holanda e Estados Unidos são os principais compradores da fruta brasileira [3]. As variedades Haden, Tommy-Atkins e Keitt estão entre os principais cultivares produzidos no país.

O sabor é principalmente uma resposta integrada às sensações do gosto e do aroma. A sensação do gosto é atribuída à presença dos compostos não voláteis nos alimentos (tais como açúcares, sais, ácidos, limonina) determinando as quatro sensações básicas descritas como doce, salgado, azedo e amargo. A sensação do aroma é bem mais complexa, pois o olfato humano pode discriminar entre milhares de compostos voláteis. São os compostos voláteis os responsáveis pelo sabor característico dos alimentos [13].

\footnotetext{
Recebido para publicação em 05/11/2001. Aceito para publicação em 12/01/2004 (000773).

2. Laboratório de Análise de Alimentos, Departamento de Ciência de Alimentos, Faculdade de Engenharia de Alimentos, UNICAMP. Caixa Postal 6121. CEP 13083-970. Campinas, S.P.

3. Instituto de Química de São Carlos, USP. São Carlos, S.P.

* A quem a correspondência deve ser enviada.
}

A revisão da literatura evidenciou que ocorre uma grande diferença entre os compostos voláteis de diferentes cultivares de manga e também da fruta procedente de diferentes regiões geográficas. As variedades Baladi e Alphonso, do Egito [4], apresentaram $\beta$-mirceno e (Z)-ocimeno no mesmo intervalo de concentração. Porém, mirceno foi o principal volátil da manga Alphonso, enquanto limoneno foi mais abundante na manga Baladi (40ppm). A variedade Alphonso procedente da Índia apresentou $(Z)$ e (E) ocimenos como os compostos majoritários [8]. Os terpenos também foram os principais voláteis de três cultivares de manga originárias do Sirilanka [10]. Uma das variedades, a Jaffna, produziu principalmente cis- $\beta$-ocimeno (38\%), enquanto as variedades Willard e Parrot, principalmente $\alpha$-terpinoleno, em porcentagem similar (35\%). Car-3-eno foi produzido em quantidade relativamente alta pela manga Parrot (12\%), mas não foi detectado na Jaffna. A manga africana caracterizou-se por apresentar um número bastante grande de compostos sesquiterpênicos [14]; sendo eremofileno o componente principal. Foram também detectados vários sesquiterpenos tricíclicos, como aromadendreno, aloaromadendreno e $\alpha$-gurjuneno. A manga australiana apresentou butanoato de etila e terpinoleno como componentes principais. A grande proporção de butanoato de etila (45\%) e de outros ésteres alifáticos não era usual em mangas [2].

Devido à grande variação observada, o presente trabalho teve como objetivo estudar os componentes voláteis de três cultivares de manga do Estado de São Paulo, com alto valor comercial. 


\section{2 - MATERIAL E MÉTODOS}

\section{1 - Seleção da matéria-prima}

As frutas procedentes do Estado de São Paulo (Monte Alto, Taquaritinga e Itápolis) foram adquiridas em caixas na CEASA de Campinas, durante a época de suas respectivas safras (dezembro, janeiro e fevereiro, referentes aos anos de 1990 e 1991). Foram analisadas assim que atingiram plena maturação, julgada pelas propriedades sensoriais de coloração da casca, textura e aroma da fruta.

\section{2 - Preparo da amostra}

A polpa da manga madura, após eliminação manual da casca e do caroço, foi cortada em cubos de aproximadamente $1 \mathrm{~cm}^{3}$ e misturada com $30 \%$ de $\mathrm{NaCl}$ p.a. (Merck). Duzentos gramas de amostra foram colocados no balão de sistema de coleta de voláteis [6]. Foram analisadas duplicatas de 3 lotes para cada cultivar, sendo que cada amostra consistiu de três frutas retiradas ao acaso de um lote de 40 frutas.

\section{3 - Isolamento dos compostos voláteis}

Para isolar os compostos voláteis foi utilizada uma técnica de headspace dinâmico, na qual os compostos voláteis foram forçados a passar através de uma minicoluna contendo polímero poroso (Porapak Q, 80-100mesh, Waters Associates), por meio de sucção [6]. As condições do condicionamento prévio do polímero e da desorção dos voláteis por eluição diferiram das do método original. Neste estudo, o polímero foi previamente condicionado a $170^{\circ} \mathrm{C}$, por no mínimo 8 horas, sob um fluxo de nitrogênio ultrapuro, que foi ainda purificado por passagem em filtros de peneira molecular. A desorção dos voláteis foi realizada por eluição dos compostos voláteis pela passagem de $300 \mu \mathrm{L}$ de hexano Lichrosolv (Merck) pelo polímero.

O tempo de coleta foi estabelecido em 2 horas, após a padronização cuidadosa das condições ótimas de captura dos compostos voláteis de manga [5].

\section{4 - Cromatografia gasosa de alta resolução}

Para separar os compostos voláteis foi utilizado um cromatógrafo a gás, marca Varian, modelo 3300, munido com detector de ionização de chama e integrador Varian, modelo 4290. As condições da análise em coluna capilar de sílica fundida $(50 \mathrm{~m}$ de comprimento por 0,21 mm de diâmetro interno) e de fase líquida SE-54, foram: hidrogênio como gás de arraste a uma velocidade linear de $50 \mathrm{~cm} / \mathrm{s}$; nitrogênio como "make up gas", a $30 \mathrm{~mL} / \mathrm{min}$; fluxos de hidrogênio e ar no detector a 30 e $300 \mathrm{~mL} / \mathrm{min}$, respectivamente; temperaturas do detector e injetor de $280^{\circ} \mathrm{C}$ e $250^{\circ} \mathrm{C}$, respectivamente. O volume injetado da amostra foi de $2 \mu \mathrm{L}$ e o tipo de injeção foi "splitless", com período de $0,75 \mathrm{~min}$ para a abertura da válvula. A temperatura da coluna foi de $50^{\circ} \mathrm{C}$ por $8 \mathrm{~min}$ e programada de $1,5^{\circ} \mathrm{C} \mathrm{min}^{-1}$ até $80^{\circ} \mathrm{C}, 3^{\circ} \mathrm{C} \mathrm{min}^{-1}$ até $130^{\circ} \mathrm{C}, 2^{\circ} \mathrm{C} \mathrm{min}^{-1}$ até $150^{\circ} \mathrm{C}$ e $5^{\circ} \mathrm{C} \mathrm{min}^{-1}$ até $200^{\circ} \mathrm{C}$.

\section{5 - Identificação dos compostos voláteis}

Os componentes voláteis foram identificados por espectrometria de massas e Índices de Kovats.

Para a espectrometria de massas, as amostras foram injetadas em um cromatógrafo a gás HewlettPackard modelo 5890, equipado com um detetor de massas HP-MSD-5970. A temperatura da coluna de sílica fundida, de $50 \mathrm{~m}$ e $0,21 \mathrm{~mm}$ de diâmetro interno e fase ligada $\mathrm{BP}-1$, foi programada a $60^{\circ} \mathrm{C}$ por $2 \mathrm{~min}$ e depois a $2^{\circ} \mathrm{C} \mathrm{min}^{-1}$ até $280^{\circ} \mathrm{C}$. Foi utilizado hélio como gás de arraste a $25 \mathrm{~cm} \mathrm{~s}^{-1}$. A temperatura do detector foi de $280^{\circ} \mathrm{C}$, a voltagem de ionização, $70 \mathrm{eV}$ e a velocidade de varredura, 10scan $\mathrm{s}^{-1}$.

Para obtenção dos Índices de Kovats, uma mistura de padrões de alcanos $\left(\mathrm{C}_{9}-\mathrm{C}_{20}\right)$ foi preparada usando-se hexano Lichrosolv (Merck) como solvente. Co-injeções da amostra e da mistura dos padrões forneceram os valores experimentais dos Índices de Kovats nas condições cromatográficas usadas por JENNINGS \& SHIBAMOTO [9] e nas condições deste estudo. Os Índices de Kovats de alguns compostos monoterpênicos puros $(\alpha$-pineno, $\beta$-pineno, $\beta$-mirceno, limoneno, $\beta$ cariofileno e $\alpha$-humuleno) foram obtidos nas condições cromatográficas utilizadas na separação analítica.

\section{6 - Análise estatística}

Diferenças na composição de voláteis entre lotes de um mesmo cultivar de manga foram verificadas através da análise dos dados (porcentagem relativa) por Análise de Variância e diferenças entre as médias foram investigadas pelo teste de Fischer (LSD).

\section{3 - RESULTADOS E DISCUSSÃO}

O uso da coluna capilar de sílica fundida de alta resolução para a separação dos componentes e de injeção "splitless" para a análise de traços permitiu a detecção de 55 componentes para a manga Haden, 55 para a Tommy-Atkins e 35 para a Keitt (Figura 1).

Foram obtidos os espectros de massas de alguns componentes voláteis de manga, que apresentaram fragmentação típica e os íons moleculares. Uma descrição detalhada da interpretação dos espectros de massas encontra-se em FRANCO [5]. Na Tabela 1 são apresentados os dados obtidos da fragmentação desses compostos.

Os valores dos Índices de Kovats obtidos neste estudo e nas condições usadas por JENNINGS \& SHIBAMOTO [9], para comparação no caso de não disponibilidade de padrões de terpenos, estão indicados na Tabela 2. As condições usadas por estes autores foram: temperatura inicial de $80^{\circ} \mathrm{C}$ e programação de $2^{\circ} \mathrm{C} \mathrm{min}^{-1}$ até $200^{\circ} \mathrm{C}$. No caso de disponibilidade de terpenos, os Índices de Kovats foram obtidos nas condições de programação de temperatura usadas neste estudo. Nas condições usadas por JENNINGS \& SHIBAMOTO [9], os componentes da amostra que eluíram antes do car-3-eno tiveram seus picos quase fundidos no cromatograma e os Índices de Kovats não puderam ser determinados. 

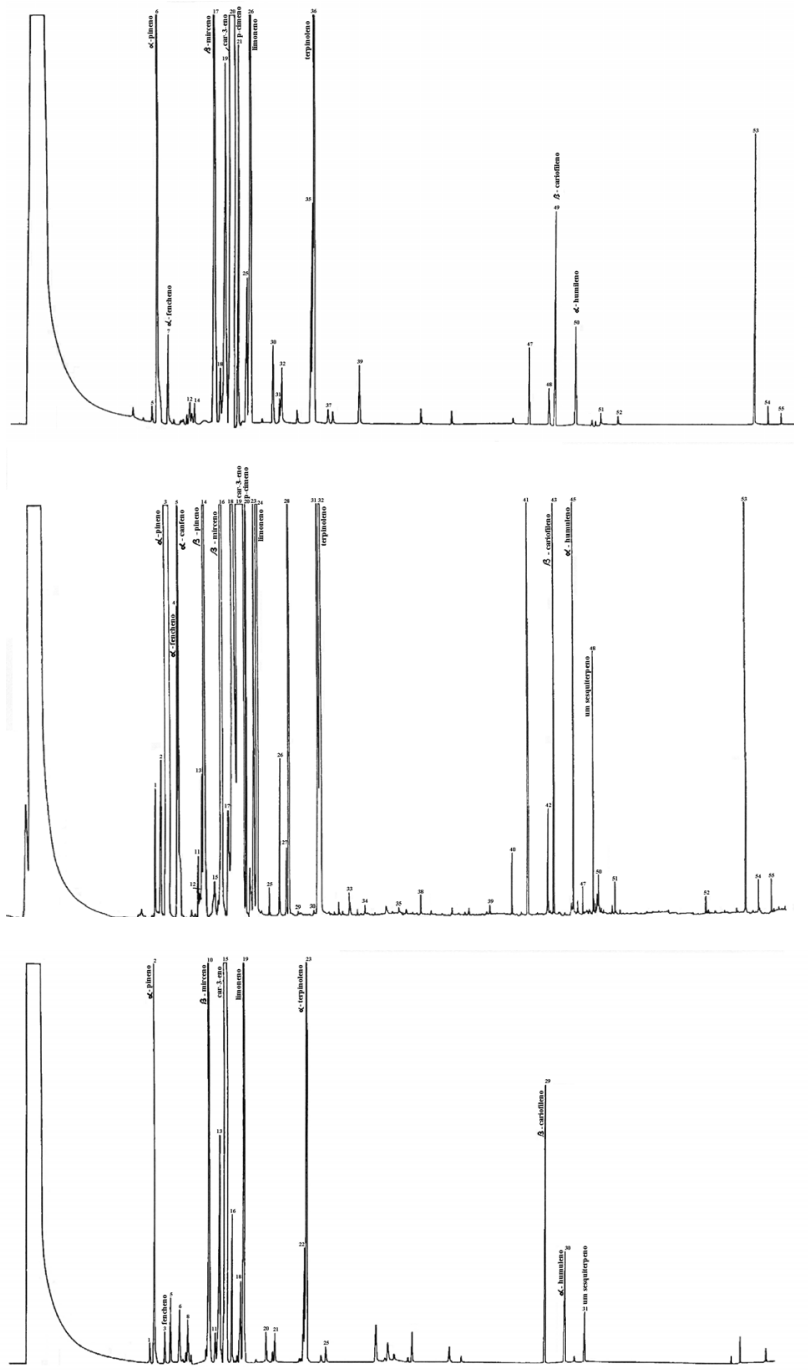

c)

FIGURA 1. Cromatogramas típicos dos compostos voláteis dos cultivares de manga Haden (a) Tommy-Atkins (b) e Keitt (c).

TABELA 1. Íons majoritários provenientes da fragmentação de alguns compostos voláteis de manga por espectrometria de massas

\begin{tabular}{ll}
\hline Compostos & \multicolumn{1}{c}{$\mathrm{m} / \mathrm{z}$ (abundância relativa) } \\
\hline$\alpha$-pineno & $93(100) 91(43) 92(42) 77(28) 79(24) 41(23) 39(22) 136(12)$ \\
$\alpha$-fencheno & $93(100) 41(70) 79(61) 39(47) 77(39) 91(38) 55(37) 92(35)$ \\
$\alpha$-canfeno & $93(100) 121(67) 41(55) 67(39) 107(38) 69(33) 91(31) 77(29)$ \\
$\beta$-pineno & $93(100) 41(55) 69(33) 91(31) 77(29) 79(26) 39(26) 94(14)$ \\
$\beta$-mirceno & $41(100) 69(74) 93(60) 39(30) 91(17) 67(16) 79(15) 55(15)$ \\
car-3-eno & $93(100) 91(50) 77(36) 92(33) 79(31) 39(26) 41(26) 136(20)$ \\
p-cimeno & $119(100) 91(36) 134(24) 93(23) 39(20) 77(20) 117(16) 65(16)$ \\
$\beta$-felandreno & $93(100) 91(41) 77(34) 79(22) 41(18) 39(18) 136(18) 94(14)$ \\
limoneno & 68(100) 67(79) 93(67) 39(39) 79(36) 41(32) 53(28) 107(2) \\
$\alpha$-terpinoleno & $93(100) 121(87) 136(68) 91(57) 79(43) 77(40) 39(34) 41(32)$ \\
$\beta$-cariofileno & $41(100) 93(68) 91(63) 79(60) 69(58) 113(54) 105(44) 39(40)$ \\
$\alpha$-humuleno & $93(100) 80(38) 41(34) 121(28) 92(25) 79(23) 91(23) 67(20)$ \\
sesquiterpeno N.I. & $41(100) 105(76) 79(66) 93(65) 91(62) 67(60) 107(60) 81(50)$ \\
\hline
\end{tabular}

N.I. não identificado.

a)
TABELA 2. Índices de Kovats de alguns compostos voláteis de manga

\begin{tabular}{|c|c|c|c|c|}
\hline \multirow{3}{*}{ Composto } & \multicolumn{4}{|c|}{ Índices de Kovats } \\
\hline & \multicolumn{2}{|c|}{ Condições de Jennings e Shibamoto ${ }^{a}$} & \multicolumn{2}{|c|}{ Condições do presente trabalho ${ }^{b}$} \\
\hline & experimental & literatura & composto desconhecido $^{d}$ & padrão ${ }^{d}$ \\
\hline$\alpha$-pineno & $--^{\circ}$ & 942 & 949 & 950 \\
\hline$\alpha$-fencheno & $--^{\circ}$ & $--^{\circ}$ & --- & --- \\
\hline$\alpha$-canfeno & $--^{c}$ & 948 & --- & --- \\
\hline$\beta$-pineno & $--^{c}$ & 981 & 981 & 984 \\
\hline$\beta$-mirceno & $--^{c}$ & 986 & 994 & 994 \\
\hline car-3-eno & 1010 & 1000 & --- & --- \\
\hline p-cimeno & 1020 & 1020 & ---- & --- \\
\hline$\beta$-felandreno & 1029 & 1025 & --- & --- \\
\hline limoneno & 1032 & 1030 & 1036 & 1038 \\
\hline$\alpha$-terpinoleno & 1095 & 1082 & 1109 & 1109 \\
\hline$\beta$-cariofileno & 1425 & 1428 & --- & ---- \\
\hline$\alpha$-humuleno & 1463 & 1465 & ---- & ---- \\
\hline sesquiterpeno N.I. & 1497 & $--^{c}$ & --- & --- \\
\hline
\end{tabular}

a Temperatura inicial de $80^{\circ} \mathrm{C}$ e programação a $2^{\circ} \mathrm{C} \min ^{-1}$ até $200^{\circ} \mathrm{C}[9] .{ }^{\circ} 50^{\circ} \mathrm{C}$ por $8 \mathrm{~min}, 1,5^{\circ} \mathrm{C}$ até $80^{\circ} \mathrm{C}, 3^{\circ} \mathrm{C} \min ^{-1}$ até $130^{\circ} \mathrm{C}, 2^{\circ} \mathrm{C} \min ^{-1}$ até $150^{\circ} \mathrm{C}, 5^{\circ} \mathrm{C} \min ^{-1}$ até $230^{\circ} \mathrm{C} .{ }^{c}$ valores não determinados ou não encontrados na literatura. ${ }^{\mathrm{d}}$ valores relatados
apenas para comparação com os padrôes disponiveis. N.I. não identificado.

As diferenças entre os cultivares foram evidenciadas nos cromatogramas típicos apresentados na Figura 1 e na Tabela 3. Os compostos voláteis identificados nos três cultivares e suas respectivas percentagens relativas estão indicados na Tabela 3. Apesar de apenas 12 compostos voláteis terem sido identificados, eles representaram, no mínimo, 97\% de contribuição à área total de cada cultivar. Os três lotes estudados de cada cultivar apresentaram grande homogeneidade, não apresentando diferença significativa a um nivel de 5\% de significância.

Os três cultivares estudados foram semelhantes entre si. Os hidrocarbonetos monoterpênicos foram os compostos dominantes, com uma contribuição de pelo menos 95\% da amostra, em todos os três cultivares. Hidrocarbonetos terpênicos também foram os compostos predominantes em outras frutas brasileiras, como umbu-cajá, camu-camu e araçá-boi [7].

TABELA 3. Compostos voláteis dos cultivares Haden, Tommy-Atkins e Keitt

\begin{tabular}{lccc}
\hline Composto & \multicolumn{3}{c}{ Porcentagem relativa $a^{a} \pm$ desvio padrão } \\
& Haden & Tommy-Atkins & Keitt \\
\hline$\alpha$-pineno & $1,5 \pm 0,1$ & $25,0 \pm 2,0$ & $1,3 \pm 0,1$ \\
$\alpha$-fencheno & $0,1 \pm 0,0$ & $0,1 \pm 0,0$ & $0,1 \pm 0,0$ \\
$\alpha$-canfeno & $\operatorname{tr}$ & $0,6 \pm 0,2$ & $\operatorname{tr}$ \\
$\beta$-pineno & $\operatorname{tr}$ & $2,6 \pm 0,1$ & $\operatorname{tr}$ \\
$\beta$-mirceno & $2,8 \pm 0,2$ & $2,1 \pm 0,1$ & $2,7 \pm 0,2$ \\
N.I. & $0,6 \pm 0,1$ & $0,6 \pm 0,0$ & $0,8 \pm 0,1$ \\
car-3-eno & $85,2 \pm 1,0$ & $60,0 \pm 2,0$ & $85,3 \pm 0,0$ \\
p-cimeno & $0,6 \pm 1,0$ & $0,5 \pm 0,0$ & $0,3 \pm 0,1$ \\
$\beta$-felandreno & $0,3 \pm 0,1$ & $0,3 \pm 0,2$ & $0,2 \pm 0,1$ \\
limoneno & $3,3 \pm 0,2$ & $3,3 \pm 0,1$ & $2,9 \pm 0,1$ \\
$\alpha$-terpinoleno & $3,2 \pm 0,2$ & $2,4 \pm 0,1$ & $2,9 \pm 0,2$ \\
$\beta$-cariofileno & $0,2 \pm 0,1$ & $0,3 \pm 0,1$ & $1,0 \pm 0,2$ \\
$\alpha$-humuleno & $0,1 \pm 0,0$ & $0,2 \pm 0,0$ & $0,5 \pm 0,1$ \\
sesquiterpeno N.I. & $\operatorname{tr}$ & $0,1 \pm 0,0$ & $0,1 \pm 0,1$ \\
\hline
\end{tabular}

${ }^{a}$ Média de 3 lotes. tr: traços $(<0,1 \%)$. N.I. não identificado. 
MACLEOD \& SNYDER [11], no seu estudo sobre a manga Keitt e Tommy-Atkins da Flórida, tinham enfatizado a semelhança entre estes dois cultivares, o que foi um resultado inesperado, considerando a grande variabilidade entre cultivares até então investigados. No presente trabalho, porém, a Haden e a Keitt mostraram maior similaridade, enquanto o cultivar TommyAtkins foi o que mais se diferenciou.

Ambos os cultivares, Haden e Keitt, tiveram 85\% de car-3-eno e $1,5 \%$ de $\alpha$-pineno, enquanto a TommyAtkins apresentou 60\% e 25\% desses dois compostos, respectivamente. A principal diferença no cromatograma entre a Keitt e a Haden ocorreu na região dos hidrocarbonetos sesquiterpênicos. A primeira apresentou $1,0 \%, 0,5 \%$ e $0,1 \%$ de $\beta$-cariofileno, $\alpha$-humuleno e o sesquiterpeno não identificado, respectivamente, enquanto a segunda apresentou $0,2 \%, 0,1 \%$ e traços desses compostos. A Tommy-Atkins, além das principais diferenças já mencionadas, apresentou uma maior porcentagem de $\alpha$-canfeno $(0,6 \%)$, de $\beta$-pineno $(2,6 \%)$ e de três compostos não identificados, numerados como pi$\cos 13,28$ e 41 neste cultivar.

MACLEOD \& SNYDER [11] obtiveram resultados semelhantes aos deste estudo, no caso da manga Tommy-Atkins da Flórida, com $60 \%$ de car-3-eno e $22,5 \%$ de $\alpha$-pineno. É interessante notar que estes autores usaram destilação-extração simultânea (SDE), enquanto neste estudo foi usada uma técnica de headspace dinâmico. A identificação de compostos de baixa e média volatilidade e a ausência de compostos de alto ponto de ebulição, tais como lactonas, identificadas em outros cultivares [8], salientou a similaridade dos resultados dos dois trabalhos. Isso é uma rara demonstração na área de voláteis, em que dois métodos diferentes, baseados em diferentes princípios, possam chegar aos mesmos resultados.

A manga Keitt do Estado de São Paulo apresentou mais car-3-eno em relação ao cultivar da Flórida, 86\% e $76 \%$, respectivamente. Esta diferença pode ser devida à prática de aceleração da maturação no Brasil, sendo as mangas Keitt colocadas em câmaras, com passagem de etileno por 48 horas, antes de serem distribuídas no comércio.

$\beta$-pineno, car-3-eno, mirceno, limoneno, p-cimeno, terpinoleno, e cariofileno, identificados neste estudo, estão entre os 13 compostos voláteis detectados nos cultivares Keitt e Tommy-Atkins da Flórida, através de um método de headspace estático, com aquecimento da amostra a $80^{\circ} \mathrm{C}$ por 15 minutos [12]. Car-3-eno foi também o composto majoritário em ambos os cultivares. A concordância dos resultados revela que estes compostos são os diretamente envolvidos no aroma da fruta, pois a técnica de headspace, dinâmica ou estática, compreende apenas os compostos voláteis em equilíbrio com a amostra, em um sistema fechado, tal como atingem a membrana nasal, enquanto SDE compreende todos os compostos voláteis presentes no alimento.

Quinze variedades de manga do Pará foram estudadas por SDE, entre elas os cultivares Haden, Tommy-
Atkins e Keith (o mesmo que Keitt), com, respectivamente, 14, 12 e 16 compostos identificados [1]. Estas apresentaram também car-3-eno como o composto majoritário, porém, a porcentagem de car-3-eno da Keitt do Pará (57\%) foi menor do que a do cultivar da Flórida (76\%). Os cultivares do Pará, no entanto, diferiram dos cultivares da Flórida e do Estado de São Paulo, principalmente em relação ao composto $\alpha$-pineno. Enquanto os cultivares Haden e Tommy-Atkins deste estudo apresentaram, respectivamente, $1,5 \%$ e $25 \%$ de $\alpha$-pineno, este composto nem foi detectado nas mangas procedentes do Pará, que apresentaram 9,2\% e 19\%, respectivamente, de um outro terpeno, tricicleno. Em relação ao cultivar Keitt, no entanto, a manga do Pará apresentou alta porcentagem de $\alpha$-pineno (20\%), enquanto a manga do Estado de São Paulo apenas 1,3\%.

Essas diferenças marcantes poderiam estar associadas à região geográfica ou então ao método utilizado. Porém, os resultados obtidos por SDE por MACLEOD \& SNYDER [11] foram semelhantes aos obtidos por headspace estático, em mangas procedentes da Flórida e, também aos obtidos por headspace dinâmico, em mangas do Estado de São Paulo, no presente trabalho. Portanto, isto favorece a hipótese das diferenças encontradas entre as mangas brasileiras ocorrerem devido principalmente aos efeitos climáticos, já que a Flórida e o Estado de São Paulo têm clima menos quente do que o do Pará.

\section{4 - CONCLUSÕES}

Os hidrocarbonetos monoterpênicos predominam no aroma dos três cultivares de manga (Haden, TommyAtkins e Keitt) do Estado de São Paulo.

O composto car-3-eno é o componente majoritário dos cultivares Haden e Keitt. O cultivar Tommy-Atkins possui dois componentes de maior abundância, car-3-eno e $\alpha$-pineno.

Há homogeneidade entre lotes do mesmo cultivar de manga.

A manga Tommy-Atkins do Estado de São Paulo apresenta alta porcentagem de $\alpha$-pineno.

\section{5 - REFERÊNCIAS BIBLIOGRÁFICAS}

[1] ANDRADE, E.H.; MAIA, J.G.S.; BZOGHBI-MDAS, G. Aroma volatile constituents of Brazilian varieties of mango fruit. J. Food Comp. Anal. v. 13, p.27-33, 2000.

[2] BARTLEY, J.P.; SCHWEDE, A. Volatile flavor components in the headspace of the Australian or Bowen mango. J. Food Sci. v. 52, p. 353-355, 360, 1987.

[3] Canal Rural (35 Net/Sky). http://www.terra.com.br/ ruralrbs/2001/05/04/008.htm.

[4] ENGEL, K.H.; TRESSL, R. Studies on the volatile components of two mango varieties. J. Agric. Food Chem. v. 31, p. 796-801, 1983.

[5] FRANCO, M.R.B. Componentes Voláteis e Sabor de Manga (Mangifera indica L.) e mamão (Carica papaya L.). Campinas, 1992. Tese (Doutorado em Ciência de Alimentos). Faculdade de Engenharia de Alimentos, Universidade Estadual de Campinas (UNICAMP). 
[6] FRANCO, M.R.B.; RODRIGUEZ-AMAYA, D.B. Trapping of soursop (Annona muricata) juice volatile on Porapak $Q$ by suction. J. Sci. Food Agric., v. 34, p. 293-299, 1983.

[7] FRANCO, M.R.B.; SHIBAMOTO, T. Volatile composition of some Brazilian fruits: umbu-cajá (Spondias citherea), camu-camu (Myrciaria dubia), araça-boi (Eugenia stipitata) and cupuaçu (Theobroma grandiflorum). J. Agric. Food Chem., v. 48, p. 1263-1265, 2000.

[8] IDSTEIN, H.; SCHREIER, P. Volatile constituents of Alphonso mango (Mangifera indica L.). Phytochem. v. 24, p. 2313-2316, 1985.

[9] JENNINGS, W.; SHIBAMOTO, T. Quantitative Analysis of Flavor and Fragrance Volatiles by Glass Capillary Gas Chromatography. Academic Press, New York, 1980. 472p.

[10] MacLEOD, A.J.; PIERIS, N.M. Comparison of the volatile components of some mango cultivars. Phytochem., v. 23, p. 361-366, 1984.

[11] MacLEOD, A.J.; SNYDER, C.H. Volatile components of two cultivars of mango from Florida. J. Agric. Food Chem. v.33, p.380-384, 1985.
[12] MALUNDO, T.M.M.; BALDWIN, E.A.; MOSHONAS, M.G.; BAKER, R.A.; SHEWFELT, R.L. Method for the rapid headspace analysis of mango (Mangifera indica L.) homogenate volatile constituents and factors affecting quantitative results. J. Agric. Food Chem. v. 45, p. 2187-2194, 1997.

[13] THOMAZINI, M.; FRANCO, M.R.B. Metodologia para análise dos constituintes voláteis do sabor. Ciênc. Tecnol. Alim. v. 34, p. 52-59, 2000.

[14] SAKHO, M.; CROUZET, J.; SECK, S. Volatile components of African mango. J. Food Sci. v. 50, p. 548-550, 1985.

\section{6 - AGRADECIMENTOS}

Este trabalho foi escrito como parte das atividades do projeto PRONEX, financiado pelo MCT-CNPq662307/1996-8 e FINEP, 41960915.00. 